\title{
MODELISATION NUMERIQUE DE LA PROPAGATION ULTRASONORE DANS DES MATÉRIAUX COMPOSITES STRATIFIÉS ANISOTROPES
}

\author{
C. POTEL et J.F. de BELLEVAL \\ LG2mS, U.T.C., Centre de Recherches, BP. 649, F-60206 Compiègne cedex, France
}

\begin{abstract}
Résumé: Cet article présente un modèle de propagation dans un multicouche périodique constitué de couches anisotropes à $90^{\circ}$ les unes avec les autres. L'utilisation de matrices de transfert qui relient les amplitudes des ondes d'une couche à l'autre permet d'obtenir les grandeurs caractérisant la propagation dont les coefficients de réflection et de transmission.
\end{abstract}

Abstract: This paper presents a model of the propagation of ultrasonic waves in a anisotropic periodically multilayered medium with one layer at $90^{\circ}$ from the next. The use of a propagator matrix method where the amplitude of one layer can be linked to that of another layer allows us to obtain magnitudes caracterizing propagation, in particular, reflexion and transmission coefficients.

\section{1 - INTRODUCTION}

L'industrie aéronautique utilise de plus en plus, en raison de leurs meilleures caractéristiques mécaniques pour un poids donné, des matériaux composites de type Carbone-Epoxy. Ils sont souvent constitués d'un assemblage de couches dans lesquelles les fibres de carbone ont toutes la même orientation, chaque couche faisant elle-même un angle donné par rapport à celles qui l'entourent. Ce sont donc des multicouches anisotropes, et la propagation des ultrasons permettant leur contrôle est très complexe. Ces dernières années, elle a fait l'objet d'un certain nombre de travaux dont ceux de Nayfeh et Chimenti [1],[2]. Le formalisme des matrices de propagation développé par Gilbert et Backus [3] est généralement utilisé : il permet d'exprimer les déplacements et les contraintes à une interface entre deux couches à partir de ceux supposés connus à l'interface entre deux autres couches. Nous avons développé sur cette base un formalisme très général sur la propagation dans des milieux stratifiés [12] dans lesquels chaque couche a une anisotropie et une épaisseur quelconque (Nayfeh s'étant limité, dans le cas le plus général qu'il ait traité, à des couches dont la symétrie est celle du système monoclinique d'axe perpendiculiare aux interfaces). Nous ne présenterons ici, pour simplifier l'exposé, qu'un cas plus simple correspondant à un problème pratique, celui pour lequel le matériau est constitué de couches orientées à $90^{\circ}$ les unes par rapport aux autres.

\section{2 - DEFINITION DU MILIEU DE PROPAGATION}

Le milieu est donc un multicouche périodique, constitué d'un empilement de $P$ "supercouches", chacune constituée de deux couches de mêmes caractéristiques et possédant une symétrie de révolution dont l'axe A, parallèle aux interfaces, tourne de $90^{\circ}$ d'une couche à l'autre (voir fígure 1). Les milieux inférieurs (2) et supérieurs (1) sont fluides et semi-infinis. Nous étudions dans ce milieu stratifié la propagation acoustique des ondes générées par une onde incidente oblique monochromatique se propageant dans le milieu 1 et contenue dans le plan $\mathrm{x}_{1} \mathrm{Ox}_{3}$.

Définissons : $\quad \mathrm{Ox}_{3}$ l'axe d'empilement des couches

$q$ le numéro de la couche dans une supercouche : $q=1$ ou 2

$\mathrm{p}$ le numéro d'une supercouche

$\mathrm{n}$ le numéro de la couche $\mathrm{n}=2$. $(\mathrm{p}-1)+\mathrm{q}$

$\mathrm{h}$ l'épaisseur des couches

$z_{n}$ la position sur l'axe $x_{3}$ de l'interface entre deux couches telle que $z_{n}=n h$.

$\omega$ la pulsation de l'onde incidente. 


\section{3 - PROPAgation dans ChaQUe COUChE}

On définit le vecteur lenteur $\overrightarrow{\mathrm{m}} \mathrm{q}$, relié au vecteur nombre d'onde ou au vecteur direction de propagation et à la vitesse de l'onde dans la couche q par la relation :

$$
\overrightarrow{\mathrm{m}}^{\mathrm{q}}=\frac{\overrightarrow{\mathrm{n}}^{\mathrm{q}}}{\mathrm{v}^{\mathrm{q}}}=\frac{\overrightarrow{\mathrm{k}}^{\mathrm{q}}}{\omega}
$$

où $\quad \overrightarrow{\mathrm{m}}^{\mathrm{q}}, \overrightarrow{\mathrm{k}}^{\mathrm{q}}$ : vecteurs lenteur et nombre d'onde dans la couche $\mathrm{q}$

$\overrightarrow{\mathrm{n}} \mathrm{q} \quad$ : direction de propagation de l'onde dans la couche $\mathrm{q}$

$\mathrm{V}^{\mathrm{q}} \quad$ : vitesse de phase de l'onde dans la couche $\mathrm{q}$

Compte-tenu de sa polarisation longitudinale, l'onde incidente engendre dans chaque couche quatre ondes numérotées par $\eta$. Dans le référentiel choisi, (voir figure 1), la loi de Snell-Descartes [10] impose que la projection du vecteur nombre d'onde (ou du vecteur lenteur) sur un plan parallèle aux interfaces soit conservée : il y a égalité des composantes parallèles à $\mathrm{x}_{1}$ des vecteurs lenteur de toutes les ondes existantes ; nous appellerons $\mathrm{m}_{1}$ cette composante.

L'équation de propagation des ondes planes peut s'écrire en fonction du vecteur des lenteurs $[5,11,13]$ :

$$
\left(c_{i j k l}^{q} \cdot{ }^{(\eta)} m_{j}^{q} \cdot{ }^{(\eta)} m_{i}^{q}-\rho \cdot \delta_{i k}\right) \cdot{ }^{(\eta)} P_{k}^{q}=0
$$

où $\quad(\eta) \vec{p}^{q},(\eta) \vec{m}^{q} \quad$ : vecteurs polarisation et lenteur de l'onde $\eta$ dans la couche $q$

$$
\begin{array}{ll}
\rho & : \text { masse volumique du matériau } \\
\mathrm{c}_{\mathrm{ij} \mathrm{q} l} & : \text { constantes élastiques de la couche } q
\end{array}
$$

D'après ce qui précède, $\mathrm{m}_{1}$ est connu $\forall \eta, \forall \mathrm{q}$, l'onde incidente étant donnée (loi de Snell-Descartes). De plus, $(\eta)_{m_{2}} q=0 \forall \eta, \forall q$. La seule composante inconnue du vecteur lenteur de l'onde $\eta$ dans la couche $q$ est donc $(\eta) \mathrm{m}_{3} \mathrm{q}$ déterminée par l'annulation du déterminant de l'équation (2). On obtient ainsi une équation bicarrée de degré 6 en $(\eta)_{\mathrm{m}_{3}} \mathrm{q}$ qui se réduit dans le cas considéré à une équation de degré 4. La résolution de celle-ci donne quatre racines deux à deux opposées qui permettent d'obtenir les quatre vecteurs lenteur correspondant à chaque onde $(\eta)$ dans la couche q. L'équation (2) permet alors de calculer ${ }^{(\eta)} \mathrm{P}_{\mathrm{k}} \mathrm{q}$ à un facteur près ; ce vecteur est ensuite normalisé $[11,13]$.

\section{4 - EQUATIONS DE TRANSMISSION D'UNE COUCHE A LA SUIVANTE}

Le passage d'une couche $n$ à une couche $n+1$ peut être effectué en exprimant les amplitudes de déplacement des quatre ondes dans chaque couche $n$ en fonction de celles dans la couche $n+1$; ce déplacement peut s'écrire [5] :

$$
{ }^{(\eta)} \vec{u}^{p, q}=(\eta) a^{p, q} \cdot(\eta) \vec{p}^{q} \cdot e^{\left.-i \omega(\eta) \vec{m}^{q} \cdot \vec{x}-t\right)}
$$

où : $(\eta) \overrightarrow{\mathbf{u}}^{\mathrm{p}, \mathrm{q}}$ : vecteur déplacement de l'onde $(\eta)$ dans la couche $n$

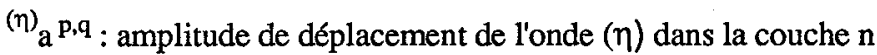

Le déplacement particulaire total est la somme des déplacements liés à chaque onde. En égalant les déplacements et les contraintes à l'interface $\mathrm{z}_{\mathrm{n}}$, on obtient une relation matricielle entre les amplitudes de la couche $\mathrm{q}=1$ en fonction de celles de la couche $\mathrm{q}=2$.

$$
\begin{aligned}
& {\left[A^{1}\right] \cdot\left[\mathcal{H}^{1}\right] \cdot\left\{\boldsymbol{A}^{p, 1}\right\}=\left[A^{2}\right] \cdot\left\{\boldsymbol{A}^{p, 2}\right\}} \\
& \text { avec }\left[\mathcal{H}^{q}\right]=\operatorname{diag}\left(e^{-i \omega \cdot(\eta) m_{3}^{q} \cdot h_{q}}\right) \text { matrice } 4 \times 4, \eta=1, \ldots, 4 \\
& \text { où : }\left\{\mathcal{A}^{\mathrm{p}, \mathrm{q}}\right\} \text { : vecteur colonne }(4 \times 1) \text { des }{ }^{(\eta)} \mathrm{a}^{\mathrm{p}, \mathrm{q}} \\
& {\left[A^{\mathrm{q}}\right] \text { : matrice } 4 \times 4 \text { donnée en annexe }}
\end{aligned}
$$


La matrice de transfert $\left[\Phi_{\mathrm{p}}\right]$ permettant d'exprimer les amplitudes de la première couche de la supercouche $p$ en fonction de celles de la première couche de la supercouche $p+1$, est obtenue en écrivant les conditions aux limites à l'interface entre deux supercouches. On en déduit $\left[\mathcal{T}_{p}\right]$ par la relation :

$$
\left[\tau_{p}\right]=\left[A^{1}\right] \cdot\left[\Phi_{p}\right] \cdot\left[A^{1}\right]^{-1}=\prod_{q=2}^{1}[A q] \cdot\left[H^{q}\right] \cdot[A q]^{-1}
$$

On peut remarquer que $\left[\mathcal{T}_{\mathrm{p}}\right]$ est le produit de matrices de propagation [4] qui dépendent uniquement des propriétés du matériau et de la fréquence $\omega$, pour chaque couche q de la supercouche $p .\left[\tau_{p}\right]$ est telle que son déterminant soit égal à 1 et que ses valeurs propres soient inverses deux à deux $[6,8,9]$. Ces quatre valeurs propres correspondent aux quatre nombres d'onde de Floquet.

Les valeurs propres $(\eta) \lambda \lambda^{q}$ de $\left[\tau_{p}\right]$, qui sont bien sûr les mêmes que celles de $\left[\Phi_{p}\right]$, sont reliées aux nombres d'onde de Floquet par:

$$
(\eta) \lambda^{q}=e^{i \omega \cdot(\eta) m_{f}^{q} \cdot h}
$$

où ${ }^{(\eta)} \mathrm{m}_{\mathrm{f}}^{\mathrm{q}}$ est la lenteur de Floquet correspondant au nombre d'onde de Floquet.

L'équation de dispersion pour un milieu multicouche périodique correspond donc à l'équation caractéristique de la matrice [ $\tau_{\mathrm{p}}$ ]. Chacune des quatre lenteurs de Floquet est associée à une onde traversant librement le matériau multicouche infini. Chacune d'elles est une combinaison des différentes ondes se propageant dans chaque couche homogène, de telle façon que les conditions limites soient satisfaites à chaque interface.

La relation entre les amplitudes de la première couche $q=1$ de la première supercouche $p=1$, et celles de la dernière couche $q=2$ de la dernière supercouche $p=P$ est donnée par:

$$
\left\{\mathcal{A}^{P, 2}\right\}=\left[\mathcal{H}^{2}\right]^{-1} \cdot\left[A^{2}\right]^{-1} \cdot\left[\Phi_{P}\right]^{P} \cdot\left\{\mathcal{A}^{1,1}\right\}
$$

\section{5 - EXEMPLES DE RESULTATS}

On suppose l'onde incidente d'amplitude égale à 1 , ce qui donne directement les coefficients de réflexion et transmisssion des ondes. Un exemple de module de coefficient de réflexion pour un milieu stratifié carbone/epoxy entouré d'eau est donné figure 2 . La propagation étant la même dans le sens $\mathrm{x}_{3}>0$ ou $\mathrm{x}_{3}<0$ les coefficients de réflexion ou de transmission sont les mêmes que l'on commence par la couche $q=1$ ou la couche $q=2$.

\section{6 - CONCLUSIONS}

Nous développons un modèle de propagation des ultrasons dans des multicouches, assemblages de couches anisotropes quelconques, dont un cas particulier plus simple est présenté dans ce papier. Ce modèle s'applique très bien à un certain nombre de matériaux en particulier une classe de composites utilisés dans l'industrie aérospatiale. Il doit permettre de mieux comprendre certains phénomènes rencontrés dans le contrôle non destructif par ultrasons de ces matériaux et d'étendre les possibilités de ces contrôles par exemple en utilisant d'autres configurations que celles utilisées actuellement (incidence oblique, optimisation de focalisation, ... ). Des expérimentations vont de plus être menées dans un proche avenir afin de mieux valider le modèle et d'adapter les constantes caractéristiques du matériau. 

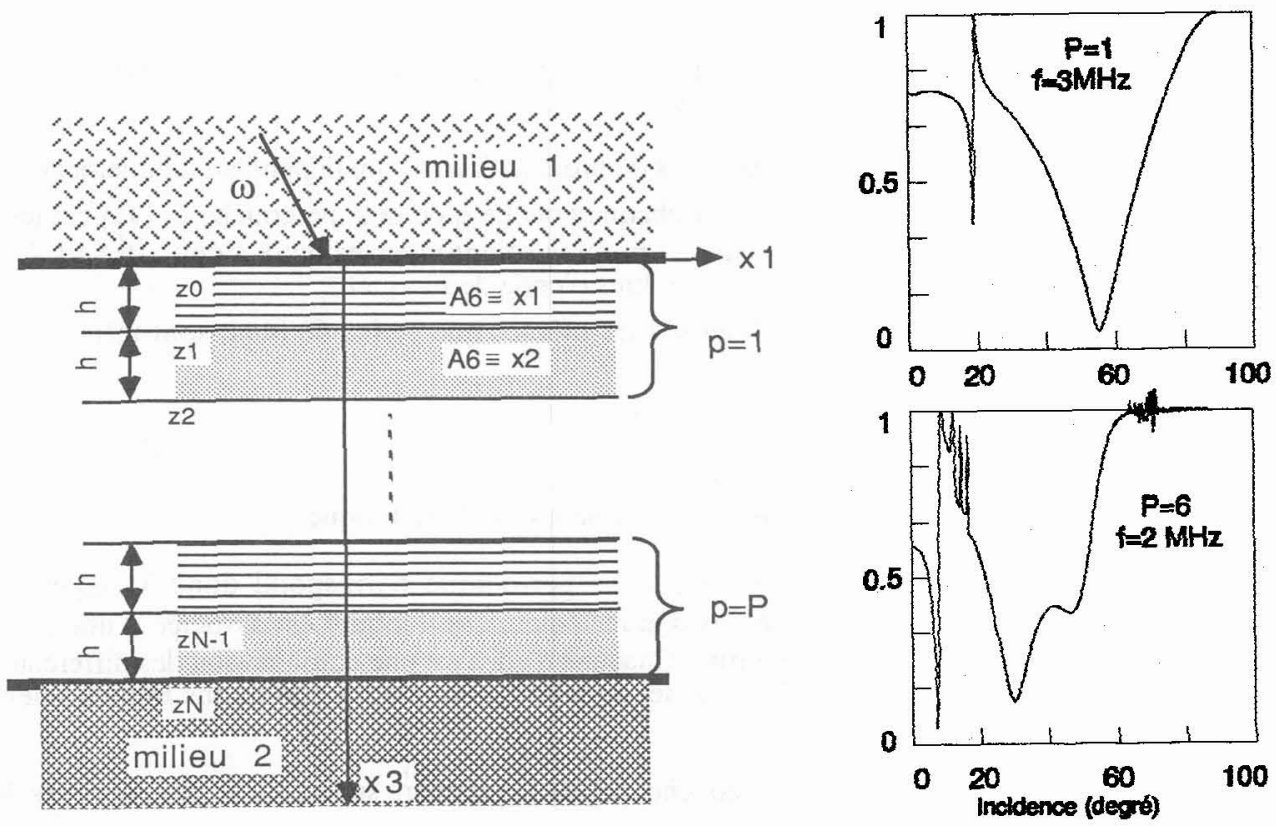

Milieu stratifié périodique

Modules des coefficients

figure 1 de réflexion pour le Carbone-Epoxy figure 2

\section{ANNEXES}

La nième colonne de la matrice $\left[\mathrm{A}^{\mathrm{q}}\right]$ s'écrit :

$$
\left\{A^{a}\right\}_{(\eta)}=\left\{\begin{array}{c}
(\eta) P_{1}^{q} \\
{ }^{(\eta)} P_{3}^{q} \\
c_{31}^{q} \cdot m_{1 \cdot}{ }_{(\eta)} P_{1}^{q}+c_{33}^{q}{ }^{(\eta)} m_{3 \cdot}^{q}{ }^{(\eta)} P_{3}^{q} \\
c_{55}^{q} \cdot\left({ }^{q} m_{3} \cdot{ }^{(\eta)} P_{1}^{q}+m_{1} \cdot{ }^{(\eta)} P_{3}^{q}\right)
\end{array}\right\}
$$

\section{BIBLIOGRAPHIE}

A.H. NAYFEH ; J. Ac. Soc. Am. 89 (4) 1991

D.E. CHIMENTI, A.H. NAYFEH ; J. of N.D.E, 9 , (2/3), 1990

F. GLBERT, GE. BACKUS ; Geophysics, 2 (31), 1986

T.C.T. TING, P. CHADWICK ; dans [7]

ROKHLIN, BOLLAND, ADLER ; J. Ac. Soc. Am. 79 (4) 1986

A.M.B. BRAGA, G. HERMANN ; dans [7]

Wave Prop. in Struc. Comp. (Am. Soc. Mec. Eng., New-York,1988), AMD 90., A.K. Mal and T.C.T TING (Eds).

M. ROUSSEAU, Ph. GATIGNOL ; Acoustica, 64, 1987

M. ROUSSEAU ; J. Ac. Soc. Am. 86 (6) 1989

E. DIEULESAINT, D. ROYER ; Ondes élastiques dans les solides ; Masson et Cie Eds

H. RIBEIRO : Thèse de doctorat U.T.C. 1992

C. POTEL, J.F. de BELLEVAL : article en préparation

H. RIBEIRO, P. LANCELEUR, J.F. de BELLEVAL : article en préparation 